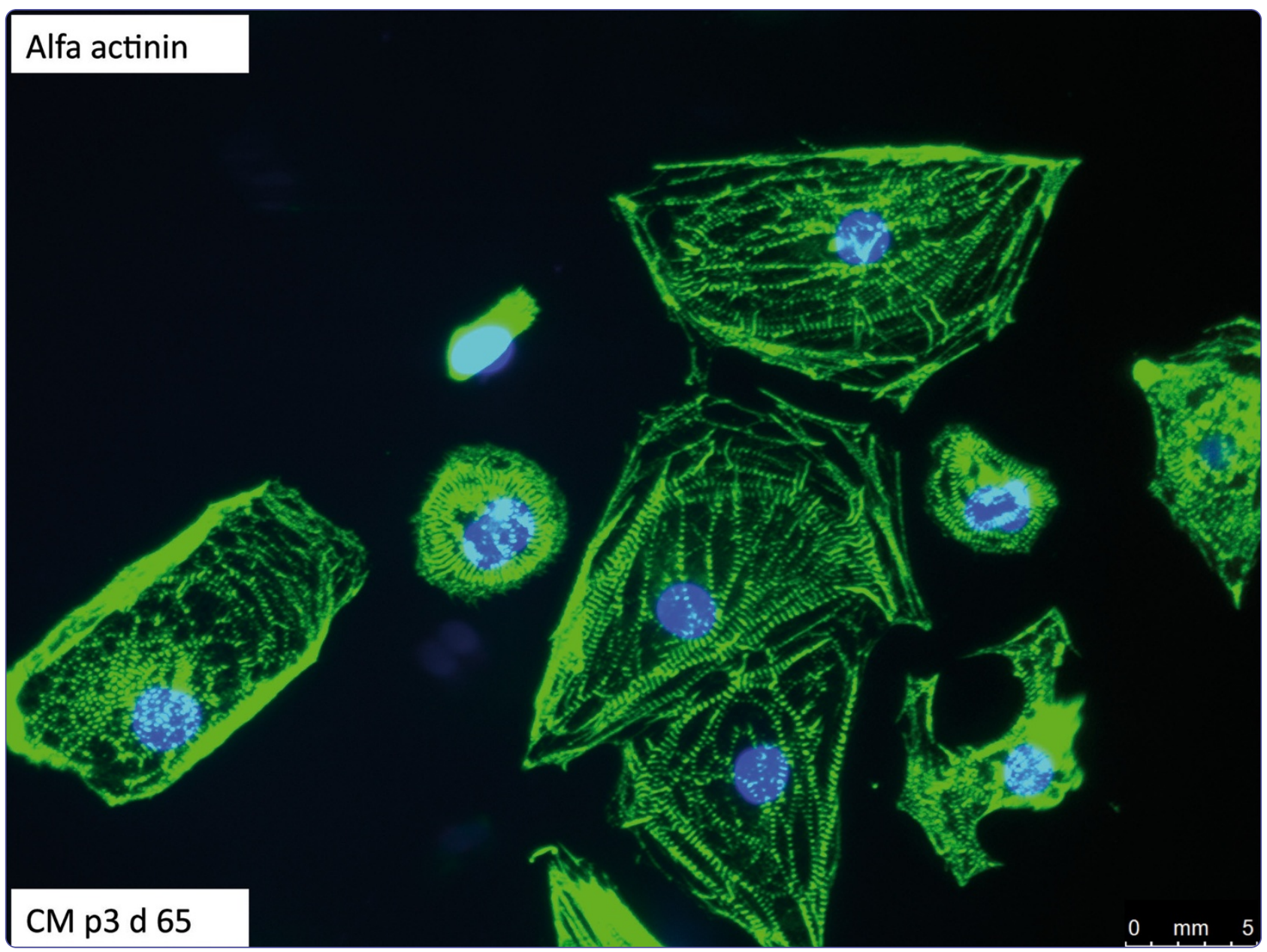

Excision of viral reprogramming cassettes by Cre protein transduction enables rapid, robust and efficient derivation of transgene-free human induced pluripotent stem cells

Kadari et al. 


\title{
Excision of viral reprogramming cassettes by Cre protein transduction enables rapid, robust and efficient derivation of transgene-free human induced pluripotent stem cells
}

\author{
Asifiqbal Kadari ${ }^{1}$, Min Lu ${ }^{2}$, Ming Li ${ }^{2,3}$, Thileepan Sekaran ${ }^{1}$, Rajkumar P Thummer ${ }^{1}$, Naomi Guyette ${ }^{2}$, Vi Chu \\ and Frank Edenhofer ${ }^{1,4^{*}}$
}

\begin{abstract}
Integrating viruses represent robust tools for cellular reprogramming; however, the presence of viral transgenes in induced pluripotent stem cells (iPSCs) is deleterious because it holds the risk of insertional mutagenesis leading to malignant transformation. Here, we combine the robustness of lentiviral reprogramming with the efficacy of Cre recombinase protein transduction to derive iPSCs devoid of transgenes. By genome-wide analysis and targeted differentiation towards the cardiomyocyte lineage, we show that transgene-free iPSCs are superior to iPSCs before Cre transduction. Our study provides a simple, rapid and robust protocol for the generation of clinical-grade iPSCs suitable for disease modeling, tissue engineering and cell replacement therapies.
\end{abstract}

\section{Introduction}

Initial reports for generating induced pluripotent stem cells (iPSCs) involved retroviruses as a mode of transgene delivery [1]. Although retroviruses are capable of reprogramming somatic cells to iPSCs, the clinical applicability of such iPSCs is limited due to the integrated transgenes carrying the risk of insertional mutagenesis [2] and tumor formation [3]. Moreover, continuous expression of transgenes in iPSCs negatively affects pluripotency [4] and limits their differentiation potential [5]. These effects have been shown by the inability to yield live chimeric mice and the diminished endodermal differentiation of iPSCs carrying transgenes [5]. Alternative approaches were explored to obtain higher efficiency with minimal genetic modifications of the cells. Various protocols circumventing viral vectors have been published, including the use of transposons [6], episomal plasmids [7], synthetic mRNA

\footnotetext{
*Correspondence: frank.edenhofer@uni-wuerzburg.de

'Stem Cell Engineering Group, Institute of Reconstructive Neurobiology, University of Bonn - Life \& Brain Center and Hertie Foundation, Sigmund-Freud Straße 25, 53105 Bonn, Germany

${ }^{4}$ Stem Cell and Regenerative Medicine Group, Institute of Anatomy and Cell Biology, Julius-Maximilians-University Würzburg, Koellikerstraße 6, D-97070 Würzburg, Germany

Full list of author information is available at the end of the article
}

[8], microRNAs [9], Sendai virus [10] as well as protein transduction [11-13]. iPSCs generated by these methods contain minimal or no genetic modifications and are generally more suitable for clinical applications than cells derived by virus-based protocols. However, still there is no gold standard for an iPSC reprogramming strategy since these non-integrating approaches exhibit limitations such as low reprogramming efficiencies, slow reprogramming kinetics, a narrow range of cell specificity, and poor reproducibility $[14,15]$. In terms of robustness and efficacy, therefore, the retroviral and lentiviral system still represents the method of choice for iPSC derivation [16].

Early attempts to improve viral-based iPSC protocols included the use of polycistronic vectors. The core element of those vectors is a cassette, consisting of cDNAs of the four transcription factors, linked together via $2 \mathrm{~A}$ selfcleaving peptide sequences $[17,18]$. This strategy allows translation of four separate polypeptides from a single mRNA strand. Thus, instead of four viruses, a single construct is sufficient to induce cellular reprogramming. This approach decreases the risk of insertional mutagenesis. Various biomedical applications of iPSCs will not strictly require cells completely free from genetic modifications. Hence, a Cre-excisable lentiviral system would provide a 
rapid and easy alternative for the generation of transgenefree iPSC clones. The usage of polycistronic vectors harboring loxP sites allows transgene excision from iPSCs via transient expression of Cre recombinase [19]. However, the reprogramming efficiency using these vectors was reported to be only $0.01 \%$ [19]. In 2009 Sommer and colleagues reported an improved lentiviral vector to overcome this limitation by yielding a reprogramming efficiency of 0.1 to $1.5 \%$ [20]. Moreover, the vector could also reprogram peripheral blood cells that are usually quite resistant towards reprogramming [21]. However, deletion of the loxP-flanked transgene cassette requires introduction of Cre recombinase activity. This activity has been accomplished by either transfection of iPSCs with a Cre-encoding plasmid $[19,22]$ or using an adenoviral Cre construct $[5,23]$ and subsequent genetic identification of successfully recombined clones. More recently, transgene-free iPSCs were obtained by excising the transgene cassette by delivery of Cre mRNA [24]. However, this protocol involves daily transfection of mRNA for a week to perform excision. This rather inefficient and laborious transfection and selection procedure makes Cre/ loxP-based iPSC derivation less appealing for obtaining transgene-free iPSCs. In fact, efficient and reliable induction of Cre recombinase activity in loxP-modified iPSCs and subsequent selection of cleaned clones represents a roadblock for the widespread use of Cre-deletable iPSC systems.

Direct delivery of biologically active Cre protein has been shown to be a highly efficient and robust method for inducing Cre recombinase activity in mammalian cells [25-28]. We reported a cell-permeable recombinant Cre protein that was generated by fusing Cre with the cell-penetrating peptide TAT and a nuclear localization sequence [29]. The TAT peptide confers cell permeability and the nuclear localization sequence targets the fusion protein to the nucleus. TAT-Cre was used for site-specific recombination in human embryonic stem cells (ESCs) with more than $90 \%$ recombination efficiency [27]. Here, we show rapid derivation of transgene-free human iPSC clones by combining direct delivery of biologically active TAT-Cre protein with robust reprogramming by a lentiviral polycistronic vector. We demonstrate that transgene deletion renders iPSCs that resemble more human ESCs with respect to gene expression than transgene-harboring cells before deletion. Moreover, we show a strong enhancement of differentiation towards the cardiac lineage in transgene-free iPSCs as compared with loxP-modified iPSCs.

\section{Materials and methods}

\section{Reprogramming of human fibroblasts and} characterization of iPSCs

The human fibroblasts used in this study were obtained from a skin punch biopsy of a 24-year-old male after obtaining informed consent and ethical clearance by the ethics committee of the University of Würzburg, Germany (ethical report number 96/11, dated 10 June 2011). Human fibroblasts were infected with the Human STEMCCA Cre-Excisable constitutive polycistronic (OKSM) lentiviral vector [22] and were seeded on irradiated mouse embryonic fibroblasts in a reprogramming medium consisting of Dulbecco's modified Eagle's medium/F12 (Sigma-Aldrich, St. Louis, MO, USA) with 20\% KnockOut Serum Replacement (Invitrogen, Carlsbad, CA, USA), $1 \mathrm{mM}$ non-animal L-glutamine (Sigma-Aldrich), $0.1 \mathrm{mM} \beta$-mercaptoethanol (Sigma-Aldrich), 1\% non-essential amino acids (Invitrogen), and $10 \mathrm{ng} / \mathrm{ml}$ basic fibroblast growth factor (Invitrogen). After 3 weeks, iPSC-like colonies were picked, expanded on matrigel-coated dishes and characterized for the pluripotency markers Oct4 (Santacruz Biotechnology, Finnell Street Dallas, Texas 75220 USA) and SSEA-4 (Millipore, Temecula, CA, USA) antibodies.

\section{TAT-Cre treatment of human iPSCs}

Human iPSCs were maintained on matrigel-coated dishes in $\mathrm{mTeSR}^{\mathrm{rm}} 1$ (STEMCELL Technologies, Temecula, CA, USA) or PluriSTEM ${ }^{\mathrm{TM}}$ (EMD Millipore, Vancouver, BC, Canada) medium. Human iPSC colonies were treated with alphazyme ( $1 \mathrm{ml} /$ well in a six-well plate; PAA, Linz, Austria) for 5 to 10 minutes to obtain single cells. Then 100,000 to 200,000 cells were seeded in each well of a six-well plate having $\mathrm{mTeSR}^{\mathrm{TM}} 1$ or PluriSTEM ${ }^{\mathrm{Ts}}$ medium with $10 \mu \mathrm{M}$ Rock inhibitor (Y27632; Sigma-Aldrich) to prevent the cell apoptosis. Twenty-four hours later, the medium was changed to $\mathrm{mTeSR}^{\mathrm{TM}} 1$ or PluriSTEM ${ }^{\mathrm{mm}}$ containing TAT-Cre (catalogue number SCR508; EMD Millipore) with different concentrations of $0.5 \mu \mathrm{M}, 1 \mu \mathrm{M}$ and $2 \mu \mathrm{M}$. Cells were incubated with TAT-Cre recombinant protein for 5 hours. Cells were grown for 1 week and colonies were expanded either monoclonally or polyclonally, and then polymerase chain reaction (PCR) was performed to assess transgene deletion. Transgene-deleted clones were expanded and characterized further by immunostaining and differentiation.

\section{Excision of the reprogramming cassette}

To confirm the transgene deletion, genomic DNA from TAT-Cre-treated subclones were isolated and PCR was performed with the following conditions: $95^{\circ} \mathrm{C}$ for $2 \mathrm{mi}$ nutes; followed by 34 cycles of $94^{\circ} \mathrm{C}$ for 30 seconds, $60^{\circ} \mathrm{C}$ for 30 seconds, and $72^{\circ} \mathrm{C}$ for 45 seconds; followed by a single cycle of $72^{\circ} \mathrm{C}$ for 5 minutes using the primers WPRE forward, ATCATGCTATTGCTTCCCGTATGGC and WPRE reverse, GGAGATCCGACTCGTCTGAGG, and $\beta$-actin forward, GGCTACAGCTTCACCACCAC and $\beta$-actin reverse, CCACCTTCCAGCAGATGTGG.

\section{Immunostaining}

For iPSC characterization, immunostaining was performed using Oct4 (1:100; Santacruz Biotechnology) and SSEA-4 (1:200; Millipore) antibodies. Briefly, cells were 
washed with phosphate-buffered saline (PBS), fixed with 4\% paraformaldehyde for 15 minutes and permeabilized in PBS containing $0.1 \%$ Triton X-100 and 5\% fetal calf serum for 30 minutes. Cells were then incubated overnight with the Oct4 and SSEA-4 antibodies. Next day, secondary antibodies Alexa 488 and Alexa 555 (1:1000; Life Technologies, Carlsbad, CA, USA) were used to detect and visualize the primary antibodies. All antibodies were diluted in blocking solution. Micrographs were taken with an Axiovert $200 \mathrm{M}$ microscope (Carl Zeiss, Oberkochen, Badenwürttemberg, Germany). The above immunostaining protocol was also performed to characterize cardiomyocytes using cardiac Troponin T (cTNT, 1:100; Abcam, Cambridge, England) and $\alpha$-actinin (1:200; Sigma-Aldrich) as a primary antibody and Alexa 488 as a secondary antibody.

\section{Teratoma formation and karyotype analysis}

Pluripotency was tested by injection of the test cell population into the testes of immune-deficient NOD/SCID mice. Then $0.5 \times 10^{6}$ to $5 \times 10^{6}$ cells were injected using a pulled glass capillary pipette that was advanced via the efferent ducts through the rete testis into the interstitial space of the testis. Animals were euthanized when palpable tumors were present or at 4 months after injection, whichever came first. The teratomas were fixed in 10\% neutral buffered formalin, dehydrated (graded alcohols), cleared (xylene) and embedded with paraffin wax (formalin fixed paraffin embedded). Then $5 \mu \mathrm{m}$ sections were cut, floated on a water bath and picked up on positively charged slides (Probe On Plus). Sections were then deparaffinized in three changes of xylene, rehydrated in a series of graded alcohols $(100 \% \times 2,70 \% \times 2,30 \% \times 2$, distilled $\mathrm{H}_{2} \mathrm{O} \times 2$ ) and hematoxylin and eosin staining was performed. Karyotype analysis was performed on 20 Gbanded metaphase cells for TAT-Cre excised human iPSC clone at p13 by Cell Line Genetics (Madiso, WI, USA).

\section{Gene expression analysis}

RNA was isolated using the RNeasy-Kit (Qiagen, Hilden, Germany). mRNA transcription levels were evaluated using the Human HT-12 array (version 4, revision 2; Qiagen, Hilden, Germany), which consists of 47,323 probes and described mRNA features. All samples were processed at least in duplicate to reduce signals arising from processing artifacts. Data processing was performed using the GenomeStudio suite version 2011.1 and the Gene expression module version 1.9.4 (both Illumina Inc., San Diego, CA, USA). Gene expression data analysis was carried out with the $\mathrm{R}$ and Bioconductor packages, and their intensities were quantile normalized. The differentially expressed genes were determined by applying the empirical Bayes test statistics, and the Benjamini-Hochberg false discovery method was used for multiple testing correction. Genes with fold-change $>2$ and $P \leq 0.5$ were considered differentially expressed and were used for subsequent analysis. The data discussed in this publication have been deposited in the National Center for Biotechnology Information Gene Expression Omnibus [GEO:GSE55725].

\section{Generation of the Cre reporter human iPSC line}

Human iPSC line del-AR1034ZIMA (del-ARiPS) clone 1.4 was treated with alfazyme to obtain a single-cell suspension. Then 200,000 cells were seeded in one well of a six-well plate in $\mathrm{mTeSR}^{\mathrm{ix}} 1$ medium with $10 \mu \mathrm{M}$ Rock inhibitor. On the next day, cells were infected with lentivirus containing $\mathrm{EF} 1 \alpha-\mathrm{Cre}$ reporter-puromycin construct (modified from original construct [30] by replacing CMV promoter by EF1 $\alpha$ promoter). Forty-eight hours later, the medium was changed to $\mathrm{mTeSR}^{\mathrm{m} 1} 1$ with puromycin $(1 \mu \mathrm{g} / \mathrm{ml})$ for 5 days to obtain colonies with stably integrated Cre reporter construct.

\section{Cardiomyocyte differentiation of human iPSCs}

Human iPSCs were maintained on matrigel-coated plates in $\mathrm{mTeSR}^{\mathrm{Tw}} 1$ medium until they reached $80 \%$ confluency. Cardiac differentiation was induced by Activin A $(5 \mathrm{ng} / \mathrm{ml})$, BMP4 $(25 \mathrm{ng} / \mathrm{ml})$ and Chir99021 $(5 \mu \mathrm{M})$ in RPMI 1640 medium containing B27 and $2 \mathrm{mM}$ glutamine as a basal medium for 3 days. From day 4, cells were kept in basal medium containing Wnt inhibitor XAV939 for 5 days followed by further culture in basal medium.

\section{Flow cytometry}

Cells $\left(1 \times 10^{6}\right)$ were trypsinized and fixed with $4 \%$ paraformaldehyde for 10 minutes. Cells were then washed with PBS, permeabilized in PBS containing 0.1\% Triton X-100 and 5\% fetal calf serum for 30 minutes and incubated for 2 hours with cTNT antibody (1:100; Abcam). No antibody was taken as a negative control. Cells were then washed once with PBS containing 0.1\% Tween-20 and resuspended in PBS containing 0.1\% Triton-X 100 and $5 \%$ fetal calf serum and secondary antibody Alexa 488 anti-mouse IgG (1:1,000; Life Technologies) for 1 hour in the dark. Finally, cells were washed again with PBS containing $0.1 \%$ Tween-20 and measured for FACS analysis. Analysis was performed by the Flow Jo program.

\section{Results}

Overview of generation of transgene-free human iPSCs with TAT-Cre application

Previous studies have shown successful derivation of transgene-free iPSCs by excising a loxP-flanked transgene cassette with Cre plasmid [19], Adeno-Cre [5,23] or Cre mRNA [24]. We aimed at improving this approach by employing Cre protein transduction to enhance the efficiency as well as to accelerate the process of obtaining transgene-free iPSCs. Our protocol does 


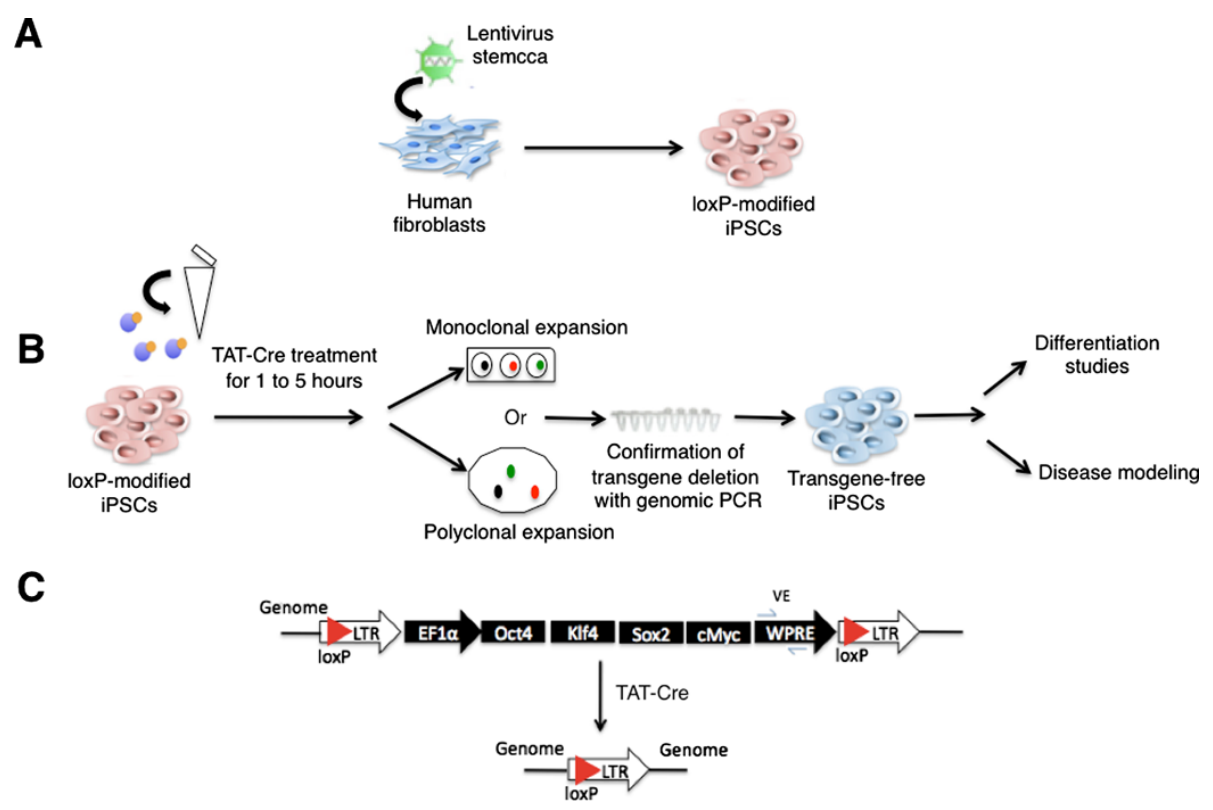

Figure 1 Scheme of induced pluripotent stem cell generation and subsequent Cre protein transduction to obtain transgene-free induced pluripotent stem cells. (A) Reprogramming of human fibroblasts to induced pluripotent stem cells (iPSCs) via lentiviral vector STEMCCA. (B) Schematic representation of TAT-Cre treatment to obtain transgene-free iPSCs. (C) Schematic representation of the genomic locus before (top) and after (bottom) Cre-mediated recombination. LTR, long terminal repeat; PCR, polymerase chain reaction.

not require repeated transfections or viral preparations (see schematic overview in Figure 1). To generate human iPSCs we utilized the lentiviral vector STEMCCA, which was shown to be more efficient [20] than earlier Cre-excisable lentiviral vectors [19]. We infected human dermal fibroblasts and obtained the AR1034ZIMA loxPmodified iPSC line (fl-ARiPS). As described in the scheme, we treated a single cell suspension of fl-ARiPS cells with a single application of TAT-Cre recombinant protein. TAT-Cre-treated cells were expanded either monoclonally or polyclonally to yield transgene-excised iPSCs ready for further analyses (Figure 1B). For the confirmation of transgene excision, PCR against the viral WPRE element was used (Figure 1C).

\section{Assessment of TAT-Cre-mediated transgene deletion efficiency}

First, we used Cre protein transduction conditions that we elaborated for Cre-mediated excision in human ESCs cultivated on mouse feeder cells [27]. Moreover, to make it practically more feasible we adopted our iPSC lines to feeder-free conditions, which we assumed to result in higher transgene deletion efficiency with a lower concentration of TAT-Cre. In particular, we prepared a single cell suspension of fl-ARiPSCs by treating them with alfazyme and seeded them on matrigel-coated plates. Twenty-four hours later, cells were treated with different concentrations of TAT-Cre for 1 to 5 hours. TAT-Cre-treated fl-ARiPS monoclones were expanded and analyzed for transgene deletion. All three monoclones analyzed that were treated with $2 \mu \mathrm{M}$ TAT-Cre showed excision of the transgene, while in the case of 0.5 and $1 \mu \mathrm{M}$ TAT-Cre treatment we observed one and two deleted clones, respectively (Figure 2A). To explore the possibility of deletion and subsequent polyclonal cell expansion, we treated the loxPmodified iPSCs with $0.5,1$ and $2 \mu \mathrm{M}$ TAT-Cre for 5 hours and expanded them polyclonally. Genomic PCR analysis revealed a faint band in the cases of 0.5 and $1 \mu \mathrm{M}$, and no band was observed after treatment with $2 \mu \mathrm{M}$ TAT-Cre, indicating a high excision efficiency that was consistent with the monoclonal analysis (Figure $2 \mathrm{~B}$ ) and previously reported results employing human ESCs [27]. In order to validate the PCR results we mixed genomic DNA from loxP-modified and transgene-deleted iPSC clones in a standardized manner representing corresponding deletion efficiencies. PCR analysis of this dilution series yielded a faint band even in the case of a 99\% mixture, while no band was observed with 100\% deleted DNA (Figure 2C). Protein transduction was repeated with seven monoclones treated with 1.5, 3 and $6 \mu \mathrm{M}$ TAT-Cre in each case. As listed in Figure 2D, all clones analyzed showed the deletion of transgenes. Increasing concentrations of TAT-Cre beyond $3 \mu \mathrm{M}$ resulted in significant cell death and affected the recovery of iPSC colonies after the treatment (data not shown). Upon using higher concentrations of TATCre, excessive cell death was prevented by shortening the time duration of TAT-Cre treatment ( $5 \mu \mathrm{M}$ TAT-Cre for 1 hour) without compromising the recombination 

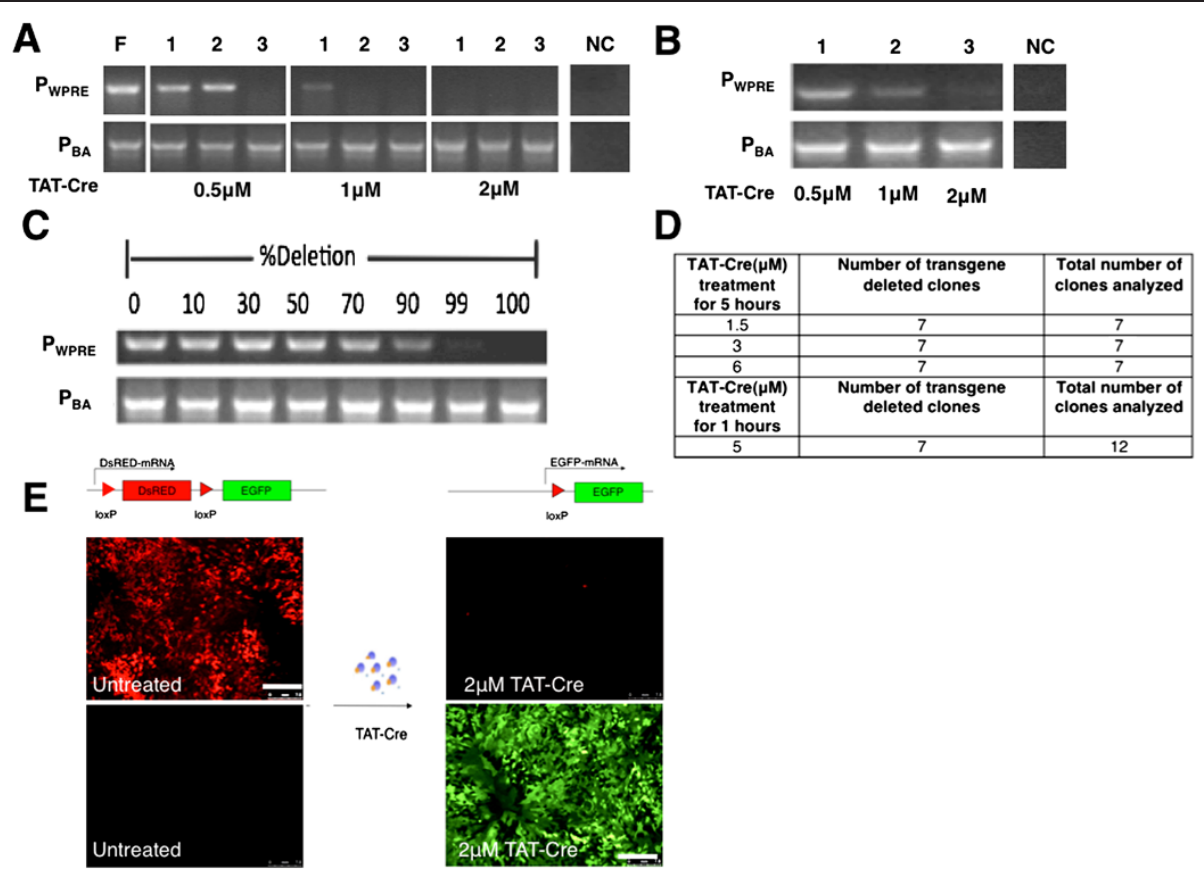

Figure 2 Assessment of TAT-Cre-mediated transgene deletion efficiency. (A) Genomic polymerase chain reaction (PCR) for the confirmation of transgene deletion. Three individual clones were treated with 0.5, 1 or $2 \mu \mathrm{M}$ TAT-Cre for 5 hours and PCR was performed using primers against WPRE and $\beta$-actin. F, AR1034ZIMA loxP-modified iPSC line clone 1 (fl-ARiPSC); NC, negative (water) control; WPRE, viral element; BA, $\beta$-actin. (B) Genomic PCR for the confirmation of transgene deletion in a polyclonal population. fl-ARiPSCs were treated with 0.5, 1 or $2 \mu \mathrm{M}$ TAT-Cre for 5 hours. Cells were expanded polyclonally and PCR was performed using primers against WPRE and $\beta$-actin. (C) Validation of genomic PCR analysis. Genomic DNA from loxP-modified and transgene deleted cells were mixed to create defined dilutions as given. (D) Quantification of transgene-deleted clones using different concentrations and time duration of TAT-Cre. (E) ARiPS-Cre reporter cell line was treated with $2 \mu \mathrm{M}$ Cre protein to validate recombination efficiency. Cre-mediated recombination induced the expression of green fluorescence protein (EGFP), by deleting the loxP-flanking RFP gene. Scale bar: $120 \mu \mathrm{m}$.

efficiency. We obtained seven transgene-free clones out of 12 clones tested (Figure 2D). To further confirm the efficiency of TAT-Cre, we monitored the recombination event by integrating a double fluorescence Cre reporter cassette through lentiviral transduction of fl-ARiPSC. We observed more than 95\% of cells showing GFP expression with $2 \mu \mathrm{M}$ TAT-Cre for 5 hours (Figure 2E), confirming the high recombination efficiency determined by PCR analyses.

\section{Characterization of transgene-free iPSCs}

To assess the pluripotency status of excised del-ARiPS clones, cells were expanded until passage 15 and stained with pluripotency-associated markers Oct4 and SSEA-4. Cells stained positive for both nuclear Oct 4 and cell surface marker SSEA-4 (Figure 3A). Furthermore, we performed genome-wide gene expression profiling on del-ARiPS cells and fl-ARiPS cells by microarray analysis. The gene expression datasets were subjected to the recently published bioinformatics assay PluriTest [31] to assess the pluripotency status of reprogrammed cells. According to this analysis, both fl-ARiPS cells and delARiPS cells cluster with human ESC line HES I3 in the red-colored background, indicating pluripotency, while fibroblasts are located in the blue region, confirming their nonpluripotent nature (Figure 3B). Notably, delARiPS cells appear slightly more shifted to the HES I3 cells as compared with fl-ARiPS cells. Expression profiling of pluripotency-associated genes Oct4, Sox2 and Rex1 as well as fibroblast genes Thy1 and Col5a2 showed similar expression pattern across the iPSCs and human ESCs. Again del-ARiPS cells appear more similar to HES I3 than fl-ARiPS cells (Figure 3C). In fact, there are 63 differentially expressed genes between HES I3 and del-ARiPS cells as compared with more than 130 genes in the case of the parental fl-ARiPS clone (Figure 3D). Furthermore, excised clone could give rise to teratoma consisting of all three germ layers, confirming its in vivo differentiation potential (Figure 3E), and showed no chromosomal aberrations as judged by karyotype analysis (Figure 3F).

\section{Improved cardiac differentiation potential of transgene-free iPSCs}

To explore whether the deletion of transgenes not only results in genome-wide transcriptional differences but 


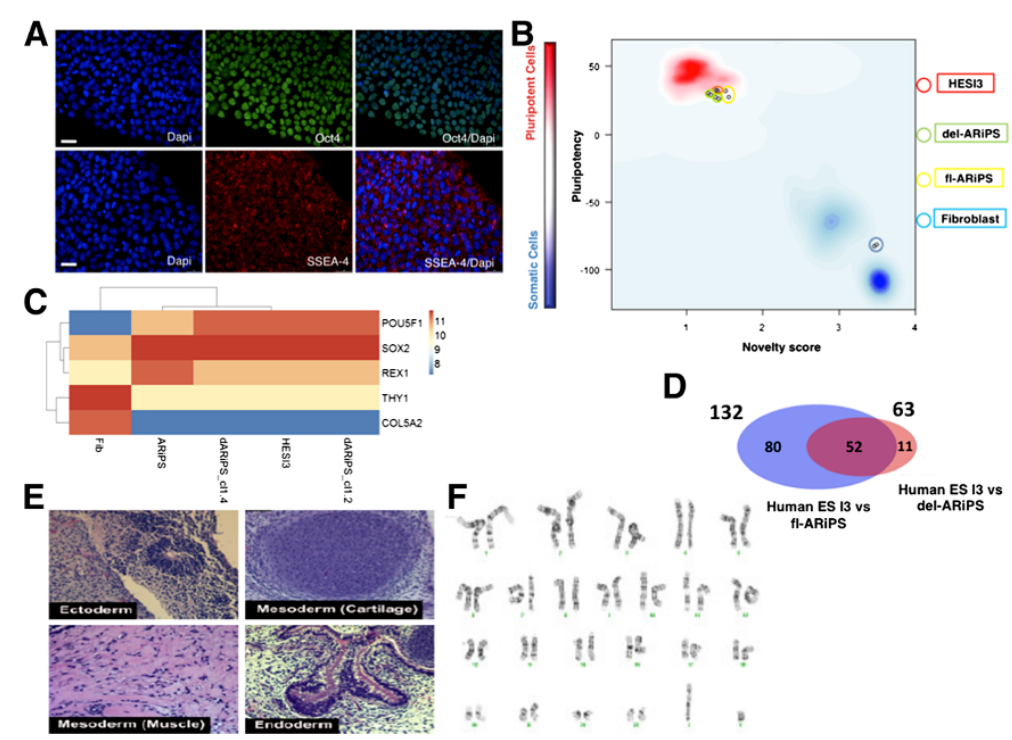

Figure 3 Characterization of transgene-free induced pluripotent stem cells. (A) Pluripotency analysis of transgene-excised clones. delARiPSCs stain positive for pluripotency-associated markers Oct4 and SSEA-4. Scale bar: 40 um. (B) PluriTest analysis of HES I3, fl-ARiPSC, del-ARiPSC cells and parental fibroblasts to assess pluripotency induction. Cells are distributed based on pluripotency and novelty scores, as indicated by color density background. Red, pluripotency; blue, nonpluripotency. (C) Heat-map representation of pluripotency and fibroblast-specific markers. Expression of pluripotency-associated markers Oct4, Sox2 and Rex1 and fibroblast specific markers Thy 1 and Col5a2 in induced pluripotent stem cells (iPSCs) and human embryonic stem cells (ESCs) used for this study. (D) Venn diagram showing differentially expressed genes. Comparison of differentially expressed genes amongst HES I3, fl-ARiPSC and del-ARiPSC cells. (E) Histological analysis of teratoma analysis of excised clone. Teratoma analysis after injection of the excised clone into SCID mice showed formation of all three germ layers: ectoderm (primitive neuroepithelium), mesoderm (cartilage, muscle) and endoderm (glands). (F) Karyotype analysis of transgene-excised clone. Cytogenetic analysis on G-banded metaphase cells from excised clone (p13) and all 20 cells demonstrated a normal male karyotype. HES I3, human ESC I3 line; fl-ARiPS, fl-ARiPS iPSC clone 1; del-ARiPS, transgene-free daughter clones of ARIPS iPSC clone 1.

also has functional consequences, we analyzed the differentiation potential by differentiation into the cardiac lineage. To achieve cardiac differentiation, we modified the protocol described by Carpenter and colleagues [32] with the inclusion of modulation of the Wnt signaling pathway using Chir99021 to enhance cardiac differentiation. We employed this protocol for the cardiac differentiation of fl-ARiPS cells and a polyclonal TAT-Cre-treated daughter cell population (2 $\mathrm{MM}$ TAT-Cre for 5 hours) (Figure 4A,B). We decided to perform polyclonal expansion in order to reduce the time duration of the entire procedure and to check whether it is possible to see the overall enhancement in differentiation capacity of a polyclonal TAT-Cre-treated population. Indeed, flow cytometry analysis using cTNT as a cardiomyocyte-specific marker indicates a strongly increased differentiation capability of del-ARiPS cells. More than $55 \%$ of differentiated del-ARiPS cells are cTNTpositive, whereas only $37 \%$ of the parental fl-ARiPSC cells stained positive for cTNT (Figure 4C).

\section{Discussion}

In this study we demonstrate an efficient way of obtaining iPSCs with minimal genetic modifications by combining the widely applied and robust lentiviral reprogramming approach with the highly efficient and less invasive TATCre-mediated transgene deletion. Earlier studies have reported the deletion of transgenes by delivering Cre as a plasmid, as Adeno-Cre, or by mRNA transfection [5,19,22-24]. The protocol elaborated by Soldner and colleagues yielded only 16 transgene-deleted clones out of 180 analyzed after transfecting iPSCs with a Cre-encoding plasmid and subsequent selection either with green fluorescent protein fluorescence or puromycin [19]. The relatively low efficiency might be due to the transient transfection of Cre, which is limiting intracellular DNA recombinase activity. Sommer and colleagues and Somers and colleagues used Adeno-Cre and Cre-IRES-Puro constructs respectively to achieve higher excision efficiencies [5,22]. A more recent study by Awe and colleagues demonstrate transgene excision in iPSC clones with putative clinical grade status using Adeno-Cre-mediated transgene deletion. During their transgene excision analysis the authors obtained only one transgene-excised subclone out of six Adeno-Cretreated clones [23]. Furthermore, deletion approaches using Cre plasmids or Adeno-Cre constructs require transfection and subsequent selection of cells with Cre recombinase activity either by flow cytometry sorting or by antibiotic selection. Such relatively complicated steps are 


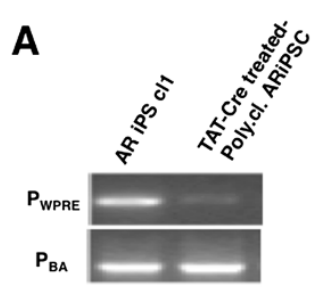

B

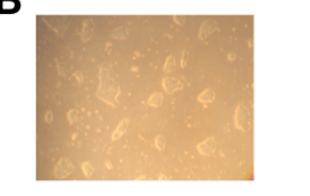

loxP-flanked or TAT-Cre treated AR iPS cl1

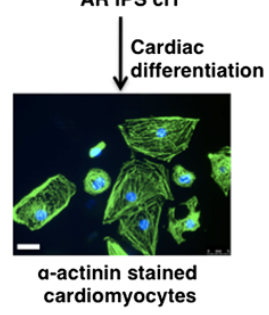

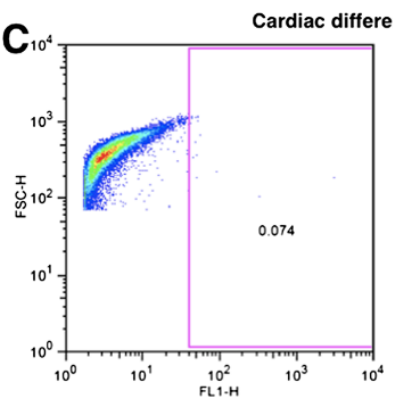

\%сTNT

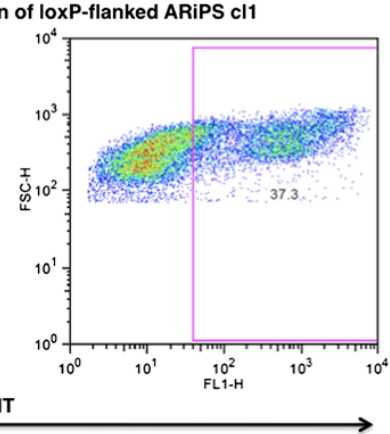

Cardiac differentiation of TAT-Cre treated polyclonal ARiPSC

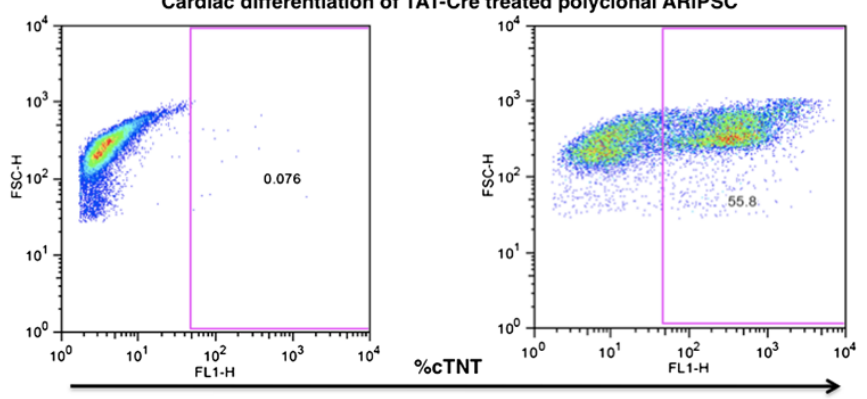

Figure 4 Improved cardiac differentiation potential of transgene-free induced pluripotent stem cells. (A) Genomic polymerase chain reaction (PCR) for the confirmation of transgene deletion. fl-ARiPS clone 1 was treated with $2 \mu \mathrm{M}$ TAT-Cre for 5 hours and PCR was performed using primers against WPRE and $\beta$-actin (BA). (B) Cardiomyocyte differentiation of iPSCs. fl-ARiPS clone 1 and its TAT-Cre-treated polyclonal cell progeny were differentiated towards cardiomyocyte lineage using cardio-inductive medium. Cells were stained with a-actinin antibody at day 15. Scale bar: $40 \mu \mathrm{m}$. (C) Flow cytometry analysis of cardiac-specific troponin T (cTNT) staining at day 15 of cardiac differentiation. Cardiac differentiation showed an increase from 37 to 56\% cTNT-positive cardiomyocytes in the case of TAT-Cre-treated cell populations. The experiment was repeated twice with similar results.

undesirable because they might be stressful to the cells [33]. Moreover, there is the possibility that Cre-encoding plasmids or viral constructs integrate into the genome [34]. More recently, the group of George Daley has reported transgene excision by transfecting loxP-modified iPSC with Cre-encoding mRNA. Notably, this procedure involves daily transfections of Cre mRNA for 4 hours up to 7 days, which again represents a complicated and stressful procedure for the cells. The protocol developed in our study, in contrast, requires just a single application of TAT-Cre recombinant protein for 5 hours due to its high recombination efficiency. By this, the use of TATCre accelerates the process of obtaining transgene-free iPSCs with minimal technical complexity. During our analysis, transgene-free iPSCs remain pluripotent. Moreover, microarray analysis indicates enhancement in the quality of iPSCs, as transgene-excised iPSCs are more similar to human ESCs with respect to gene expression profiling, confirming previous studies [19]. In fact, transgene-free iPSCs exhibit an improved differentiation potential [5]. In our study, we show enhanced cardiac differentiation of TAT-Cre-treated polyclonal iPSCs. By this we show an improvement in differentiation capacity of a polyclonal cell population after the removal of transgenes with Cre- mediated recombination. This makes TAT-Cre protein an attractive tool to obtain transgene-free iPSCs even in a polyclonal manner as recently suggested [35], circumventing the laborious selection procedure of transgeneexcised clones. In conclusion, our study provides a simple, rapid and robust protocol for the generation of superior transgene-free iPSCs suitable for disease modeling, drug and toxicity screening, as well as cell replacement therapies.

\section{Conclusions}

In summary, our study outlines efficient derivation of factor-free human iPSC lines by combinatorial use of the robust lentivirus human STEMCCA vector and highly efficient TAT-Cre protein transduction. We have shown enhanced quality of transgene-free iPSCs using microarray analysis and cardiac differentiation. Moreover, we show polyclonal expansion of transgene-deleted clones that circumvents laborious selection procedures and time-consuming analysis of subclones.

\section{Abbreviations}

CTNT: cardiac Troponin T; del-ARiPS: AR1034ZIMA transgene-free daughter clones of ARIPS iPSC clone 1; fl-ARiPS: AR1034ZIMA loxP-flanked transgene ARiPS iPSC clone 1; HES I3: human ESC I3 line; ESC: embryonic stem cell; 
iPSC: induced pluripotent stem cell; PBS: phosphate-buffered saline; PCR: polymerase chain reaction.

\section{Competing interests}

MLu, MLi, NG and VC are salaried employees at EMD Millipore. The remaining authors declare that they have no competing interests.

\section{Authors' contributions}

AK was responsible for the conception, design, collection and/or assembly of data, data analysis, interpretation, manuscript writing, and final approval of the manuscript. MLu, MLi, NG, VC and TS collected and/or assembled data. RPT performed data analysis and manuscript writing. FE was responsible for conception and design, financial support, collection and/or assembly of data, data analysis and interpretation, manuscript writing, and final approval of manuscript. All authors contributed to the final draft of the manuscript.

\section{Acknowledgments}

The authors thank Gustavo Mostoslavsky (Boston University School of Medicine, USA) for providing the STEMCCA construct and Sarah Kittel-Schneider as well as Andreas Reif (Klinik und Poliklinik für Psychiatrie, Würzburg, Germany) for providing human fibroblasts. They would also like to thank Andrea Hofmann for helping to obtain gene expression data. The authors are indebted to Martina Gebhardt, Kathrin Vogt and Nicole Russ for excellent technical support and to all members of the Stem Cell Engineering Group for support and valuable discussions and critical reading of the manuscript. This work was supported by grants from the Deutsche Forschungsgemeinschaft DFG (ED79/1-2) and the German Ministry of Education and Research, BMBF (01 GN 0813) and by Merck KGaA, Darmstadt, Germany.

\section{Author details}

${ }^{1}$ Stem Cell Engineering Group, Institute of Reconstructive Neurobiology, University of Bonn - Life \& Brain Center and Hertie Foundation, Sigmund-Freud Straße 25, 53105 Bonn, Germany. ${ }^{2}$ EMD-Millipore, Bioscience Division, Stem Cell Group, Temecula, CA 92590, California. ${ }^{3}$ Present address: Irvine Pharmaceutical Services, Irvine, CA 92618, USA. ${ }^{4}$ Stem Cell and Regenerative Medicine Group, Institute of Anatomy and Cell Biology, Julius-Maximilians-University Würzburg, Koellikerstraße 6, D-97070 Würzburg, Germany.

Received: 25 September 2013 Revised: 19 December 2013 Accepted: 12 March 2014 Published: 8 April 2014

\section{References}

1. Takahashi K, Yamanaka S: Induction of pluripotent stem cells from mouse embryonic and adult fibroblast cultures by defined factors. Cell 2006, 126:663-676.

2. Mikkers $H$, Berns $A$ : Retroviral insertional mutagenesis: tagging cancer pathways. Adv Cancer Res 2003, 88:53-99.

3. Okita K, Ichisaka T, Yamanaka S: Generation of germline-competent induced pluripotent stem cells. Nature 2007, 448:313-317.

4. Kopp $J$, Ormsbee BD, Desler M, Rizzino A: Small increases in the level of Sox2 trigger the differentiation of mouse embryonic stem cells. Stem Cells 2008, 26:903-911.

5. Sommer CA, Sommer AG, Longmire TA, Christodoulou C, Thomas DD, Gostissa M, Alt FW, Murphy GJ, Kotton DN, Mostoslavsky G: Excision of reprogramming transgenes improves the differentiation potential of iPS cells generated with a single excisable vector. Stem Cells 2010, 28:64-74.

6. Kaji K, Norrby K, Paca A, Mileikovsky M, Mohseni P, Woltjen K: Virus-free induction of pluripotency and subsequent excision of reprogramming factors. Nature 2009, 458:771-775.

7. Chou BK, Mali P, Huang X, Ye Z, Dowey SN, Resar LM, Zou C, Zhang YA, Tong J, Cheng L: Efficient human iPS cell derivation by a non-integrating plasmid from blood cells with unique epigenetic and gene expression signatures. Cell Res 2011, 21:518-529.

8. Warren L, Manos PD, Ahfeldt T, Loh YH, Li H, Lau F, Ebina W, Mandal PK, Smith ZD, Meissner A, Daley GQ, Brack AS, Collins JJ, Cowan C, Schlaeger TM, Rossi DJ: Highly efficient reprogramming to pluripotency and directed differentiation of human cells with synthetic modified mRNA. Cell Stem Cell 2010, 7:618-630.

9. Anokye-Danso F, Trivedi CM, Juhr D, Gupta M, Cui Z, Tian Y, Zhang Y, Yang W, Gruber PJ, Epstein JA, Morrisey EE: Highly efficient miRNA-mediated reprogramming of mouse and human somatic cells to pluripotency. Cell Stem Cell 2011, 8:376-388.

10. Fusaki N, Ban H, Nishiyama A, Saeki K, Hasegawa M: Efficient induction of transgene-free human pluripotent stem cells using a vector based on Sendai virus, an RNA virus that does not integrate into the host genome. Proc Jpn Acad Ser B Phys Biol Sci 2009, 85:348-362.

11. Bosnali M, Edenhofer $F$ : Generation of transducible versions of transcription factors Oct4 and Sox2. Biol Chem 2008, 389:851-861.

12. Kim D, Kim CH, Moon Jl, Chung YG, Chang MY, Han BS, Ko S, Yang E, Cha KY, Lanza R, Kim KS: Generation of human induced pluripotent stem cells by direct delivery of reprogramming proteins. Cell Stem Cell 2009, 4:472-476.

13. Zhou H, Wu S, Joo JY, Zhu S, Han DW, Lin T, Trauger S, Bien G, Yao S, Zhu Y, Siuzdak G, Schöler HR, Duan L, Ding S: Generation of induced pluripotent stem cells using recombinant proteins. Cell Stem Cell 2009, 4:381-384.

14. Gonzalez F, Boue S, Izpisua Belmonte JC: Methods for making induced pluripotent stem cells: reprogramming a la carte. Nat Rev Genet 2011, 12:231-242.

15. Worsdorfer $P$, Thier M, Kadari A, Edenhofer F: Roadmap to cellular reprogramming - manipulating transcriptional networks with DNA, RNA, proteins and small molecules. Curr Mol Med 2011, 13:868-878.

16. Sommer CA, Mostoslavsky G: Experimental approaches for the generation of induced pluripotent stem cells. Stem Cell Res Ther 2010, 1:26.

17. Carey BW, Markoulaki S, Hanna J, Saha K, Gao Q, Mitalipova M, Jaenisch R: Reprogramming of murine and human somatic cells using a single polycistronic vector. Proc Natl Acad Sci U S A 2009, 106:157-162.

18. Szymczak AL, Workman CJ, Wang Y, Vignali KM, Dilioglou S, Vanin EF, Vignali DA: Correction of multi-gene deficiency in vivo using a single 'self-cleaving' 2A peptide-based retroviral vector. Nat Biotechnol 2004, 22:589-594.

19. Soldner F, Hockemeyer D, Beard C, Gao Q, Bell GW, Cook EG, Hargus G, Blak A, Cooper O, Mitalipova M, Isacson O, Jaenisch R: Parkinson's disease patient-derived induced pluripotent stem cells free of viral reprogramming factors. Cell 2009, 136:964-977.

20. Sommer CA, Stadtfeld M, Murphy GJ, Hochedlinger K, Kotton DN, Mostoslavsky G: Induced pluripotent stem cell generation using a single lentiviral stem cell cassette. Stem Cells 2009, 27:543-549.

21. Staerk J, Dawlaty MM, Gao Q, Maetzel D, Hanna J, Sommer CA, Mostoslavsky $\mathrm{G}$, Jaenisch R: Reprogramming of human peripheral blood cells to induced pluripotent stem cells. Cell Stem Cell 2010, 7:20-24.

22. Somers A, Jean JC, Sommer CA, Omari A, Ford CC, Mills JA, Ying L, Sommer AG, Jean JM, Smith BW, Lafyatis R, Demierre MF, Weiss DJ, French DL, Gadue P, Murphy GJ, Mostoslavsky G, Kotton DN: Generation of transgene-free lung disease-specific human induced pluripotent stem cells using a single excisable lentiviral stem cell cassette. Stem Cells 2010, 28:1728-1740.

23. Awe JP, Lee PC, Ramathal C, Vega-Crespo A, Durruthy-Durruthy J, Cooper A, Karumbayaram S, Lowry WE, Clark AT, Zack JA, Sebastiano V, Kohn DB, Pyle AD, Martin MG, Lipshutz GS, Phelps PE, Pera RA, Byrn JA: Generation and characterization of transgene-free human induced pluripotent stem cells and conversion to putative clinical-grade status. Stem Cell Res Ther 2013, 4:87.

24. Loh YH, Yang JC, De Los Angeles A, Guo C, Cherry A, Rossi DJ, Park $H_{\text {, }}$ Daley GQ: Excision of a viral reprogramming cassette by delivery of synthetic Cre mRNA. Curr Protoc Stem Cell Biol 2012, Chapter 4:Unit4A 5.

25. Patsch C, Edenhofer F: Conditional mutagenesis by cell-permeable proteins: potential, limitations and prospects. Handb Exp Pharmacol 2007, 178:203-232.

26. Haupt S, Edenhofer F, Peitz M, Leinhaas A, Brustle O: Stage-specific conditional mutagenesis in mouse embryonic stem cell-derived neural cells and postmitotic neurons by direct delivery of biologically active Cre recombinase. Stem Cells 2007, 25:181-188.

27. Nolden L, Edenhofer F, Haupt S, Koch P, Wunderlich FT, Siemen H, Brustle $\mathrm{O}$ : Site-specific recombination in human embryonic stem cells induced by cell-permeant Cre recombinase. Nat Methods 2006, 3:461-467.

28. Peitz M, Jager R, Patsch C, Jager A, Egert A, Schorle H, Edenhofer F: Enhanced purification of cell-permeant Cre and germline transmission after transduction into mouse embryonic stem cells. Genesis 2007, 45:508-517.

29. Peitz M, Pfannkuche $K$, Rajewsky $K$, Edenhofer F: Ability of the hydrophobic FGF and basic TAT peptides to promote cellular uptake of recombinant Cre recombinase: a tool for efficient genetic engineering of mammalian genomes. Proc Natl Acad Sci U S A 2002, 99:4489-4494. 
30. Russ $H A, B a r ~ Y$, Ravassard $P$, Efrat $S$ : In vitro proliferation of cells derived from adult human beta cells revealel by cell lineage tracing. Diabetes 2008, 57:1575-1583.

31. Muller FJ, Schuldt BM, Williams R, Mason D, Altun G, Papapetrou EP, Danner S, Goldmann JE, Herbst A, Schmidt NO, Aldenhoff JB, Laurent LC, Loring JF: A bioinformatic assay for pluripotency in human cells. Nat Methods 2011 8:315-317.

32. Carpenter L, Carr C, Yang CT, Stuckey DJ, Clarke K, Watt SM: Efficient differentiation of human induced pluripotent stem cells generates cardiac cells that provide protection following myocardial infarction in the rat. Stem Cells Dev 2012, 21:977-986.

33. Moran DM, Shen H, Maki CG: Puromycin-based vectors promote a ROS-dependent recruitment of PML to nuclear inclusions enriched with HSP70 and Proteasomes. BMC Cell Biol 2009, 10:32.

34. Glover DJ, Lipps HJ, Jans DA: Towards safe, non-viral therapeutic gene expression in humans. Nat Rev Genet 2005, 6:299-310.

35. Willmann CA, Hemeda H, Pieper LA, Lenz M, Qin J, Joussen S, Sontag S, Wanek P, Denecke B, Schuler HM, Zenke M, Wagner W: To clone or not to clone? Induced pluripotent stem cells can be generated in bulk culture. PLoS One 2013, 8:e65324.

doi:10.1186/scrt435

Cite this article as: Kadari et al.: Excision of viral reprogramming cassettes by Cre protein transduction enables rapid, robust and efficient derivation of transgene-free human induced pluripotent stem cells. Stem Cell Research \& Therapy 2014 5:47.

\section{Submit your next manuscript to BioMed Central and take full advantage of:}

- Convenient online submission

- Thorough peer review

- No space constraints or color figure charges

- Immediate publication on acceptance

- Inclusion in PubMed, CAS, Scopus and Google Scholar

- Research which is freely available for redistribution 